Journal for ImmunoTherapy of Cancer

\section{Tumor infiltrating lymphocytes (TIL) therapy in metastatic melanoma: boosting of neoantigen-specific $T$ cell reactivity and long-term follow-up}

To cite: van den Berg JH, Heemskerk B, van Rooij N, et al. Tumor infiltrating lymphocytes (TIL) therapy in metastatic melanoma: boosting of neoantigen-specific $T$ cell reactivity and long-term followup. Journal for ImmunoTherapy of Cancer 2020;8:e000848. doi:10.1136/jitc-2020-000848

- Additional material is published online only. To view please visit the journal online (http://dx.doi.org/10.1136/jitc2020-000848).

Accepted 23 June 2020

Check for updates

(C) Author(s) (or their employer(s)) 2020. Re-use permitted under CC BY-NC. No commercial re-use. See rights and permissions. Published by BMJ.

For numbered affiliations see end of article.

Correspondence to John B A G Haanen; j.haanen@nki.nl

\section{ABSTRACT}

Treatment of metastatic melanoma with autologous tumor infiltrating lymphocytes (TILs) is currently applied in several centers. Robust and remarkably consistent overall response rates, of around $50 \%$ of treated patients, have been observed across hospitals, including a substantial fraction of durable, complete responses.

Purpose Execute a phase I/II feasibility study with TIL therapy in metastatic melanoma at the Netherlands Cancer Institute, with the goal to assess feasibility and potential value of a randomized phase III trial.

Experimental Ten patients were treated with TIL therapy. Infusion products and peripheral blood samples were phenotypically characterized and neoantigen reactivity was assessed. Here, we present long-term clinical outcome and translational data on neoantigen reactivity of the $T$ cell products.

Results Five out of 10 patients, who were all anti-PD-1 naïve at time of treatment, showed an objective clinical response, including two patients with a complete response that are both ongoing for more than 7 years. Immune monitoring demonstrated that neoantigen-specific T cells were detectable in TIL infusion products from three out of three patients analyzed. For six out of the nine neoantigenspecific T cell responses detected in these TIL products, T cell response magnitude increased significantly in the peripheral blood compartment after therapy, and neoantigen-specific T cells were detectable for up to 3 years after TIL infusion.

Conclusion The clinical results from this study confirm the robustness of TIL therapy in metastatic melanoma and the potential role of neoantigen-specific T cell reactivity. In addition, the data from this study supported the rationale to initiate an ongoing multicenter phase III TIL trial.

\section{INTRODUCTION}

Melanoma is widely recognized as one of the most immunogenic human cancer types and a strong correlation between the infiltration of $\mathrm{T}$ cells, both in primary lesions and in melanoma metastases, and clinical outcome has been described. ${ }^{12}$ Among the tumorinfiltrating lymphocytes (TILs) in this disease, $\mathrm{T}$ cells with reactivity against melanomaassociated antigens and cancer-testis antigens have been observed. ${ }^{3-6}$ In addition, recent work has shown that $\mathrm{T}$ cell reactivity against the neoantigens, which are formed as a consequence of DNA damage is common in melanoma, ${ }^{7-9}$ an observation that is consistent with the high mutational burden of this disease. ${ }^{10}$ Based on the correlation between mutational burden and response to immune checkpoint blockade observed in several studies, ${ }^{11} 12 \mathrm{~T}$ cell reactivity against neoantigens is assumed to play a significant role in disease control. Collectively, metastatic melanoma is a prime candidate for cancer immunotherapy, aiming to restore tumor control by the antigen-specific $\mathrm{T}$ cell compartment.

Over the past years, treatment options for metastatic melanoma patients have significantly improved, especially by the introduction of both targeted therapies (oral BRAF plus MEK inhibitors) for BRAF V600 mutated melanomas and immune checkpoint inhibitors, such as anti- cytotoxic T-lymphocyte-associated protein 4 (CTLA$4),{ }^{13}$ anti-programmed cell death protein 1 (PD-1) ${ }^{14}$ or their combination. ${ }^{15}$ In a three arm randomized controlled phase III trial, with 945 patients, the overall 5-year survival rate was $26 \%$ for anti-CTLA-4, $44 \%$ for anti-PD-1 and $52 \%$ for the combination of both checkpoint inhibitors. ${ }^{16}$ A proportion of patients treated with these checkpoint 
inhibitors have durable remissions. ${ }^{16}$ Nevertheless, a large fraction of patients does progress on checkpoint inhibition, and hence a significant unmet medical need remains, especially for BRAF wildtype melanomas.

In parallel to the clinical development of immune checkpoint blockade, pioneering work by the Rosenberg group at the National Institutes of Health (NIH) Surgery Branch (SB) and subsequent work by, among others, Sheba Medical Center has revealed the value of adoptive cell therapy using ex vivo expanded TILs, in particular in melanoma. ${ }^{1718}$ In this process, TIL populations are generated from single cell suspensions or tumor fragments from freshly resected tumor material of melanoma patients, through an initial expansion phase in the presence of interleukin-2 (IL-2). Subsequently, the cultured TIL are expanded to very large numbers (approximately $1 \times 10^{10}$ to $1 \times 10^{11}$ cells) in a 14 day rapid expansion protocol (REP), thereby yielding the cell product that is used for infusion. No enrichment for tumor reactivity is included in this so-called 'young' TIL protocol. ${ }^{19}$ Prior to infusion of the resulting TIL products, patients are treated with lymphodepleting, but non-myeloablative, chemotherapy and following intravenous administration of the cell product, high-dose (HD) bolus IL-2 infusions are given to support the growth and survival of the infused T cells. On average, TIL therapy has shown clinical responses in approximately $50 \%$ of treated individuals, mostly in anti-PD-1 treatment naïve patients, with durable complete remissions (CR) in $10 \%-15 \%$ of patients with treatment-refractory metastatic melanoma. ${ }^{2021}$ Importantly, the encouraging results initially generated by the SB and Sheba Medical Center have been reproduced by other centers, including MD Anderson Cancer Center in Houston (48\% response rate in 31 patients) ${ }^{22}{ }^{23}$ and the Center of Cancer Immune Therapy (CCIT), Herlev Hospital, Copenhagen (42\% response rate in 25 patients). ${ }^{24}$ More recent observed response rates in patients refractory to anti-PD-1 treatment are $30 \%-40 \% .^{25}$

With the aim to potentially execute a randomized phase III trial of TIL therapy, the Netherlands Cancer Institute (NKI) initiated a phase I/II feasibility study with TIL therapy in metastatic melanoma starting in 2011 to assess feasibility and clinical activity. Here, we present the long-term clinical outcome of this study. In addition, we provide insight into the occurrence of neoantigen-specific $\mathrm{T}$ cell reactivity in the infusion product and the subsequent emergence of neoantigen-specific $\mathrm{T}$ cell responses in peripheral blood on therapy.

\section{MATERIALS AND METHODS \\ Patients}

Patients $\geq 18$ years with metastatic, cutaneous, stage IIIc/ IV melanoma, with at least one resectable metastatic lesion of sufficient size ( $\geq 3 \mathrm{~cm}$ in diameter), were eligible. Inclusion criteria included a clinical performance status $\leq 1$, absolute neutrophil count $\geq 1.5 \times 10^{9} / \mathrm{L}$, platelet count $\geq 100 \times 10^{9} / \mathrm{L}$, hemoglobin $>5 \mathrm{mmol} / \mathrm{L}$, serum Alanine
(Amino) Transaminase/Aspartate Aaminotransferase (ALAT/ASAT) $<3$ times the upper $d$ of normal, unless patients had liver metastases $(<5$ times ULN) and serum creatinine clearance of $50 \mathrm{~mL} / \mathrm{min}$ or higher. In addition, patients needed to be seronegative for HIV, hepatitis B antigen, hepatitis $\mathrm{C}$ antibody and syphilis. Exclusion criteria did not allow participation of patients with autoimmune disease, requirement of corticosteroids, two or more CNS metastases, symptomatic CNS metastasis and inability to receive HD IL-2.

All patients provided informed consent before enrolment. This study was registered as 2010-021885-31.

\section{Trial design}

Patients underwent surgical resection of a melanoma metastasis to grow TIL in vitro (see below). Prior to infusion of the TIL product, patients received a nonmyeloablative, lymphocyte-depleting preparative regimen consisting of cyclophosphamide $(60 \mathrm{mg} / \mathrm{kg} /$ day x 2 days intravenous) and fludarabine $\left(25 \mathrm{mg} / \mathrm{m}^{2} /\right.$ day x 5 days intravenous). Intravenous adoptive transfer of TILs was followed by HD intravenous IL-2 (Proleukin) (600 000 $\mathrm{IU} / \mathrm{kg} /$ dose every 8 hours up to tolerance or up to 15 doses). Supportive care consisted of blood or platelet transfusions, which were given until spontaneous hematopoietic recovery.

A first complete assessment of evaluable lesions was conducted 6 weeks after cell infusion and periodically afterwards, at 12 and 18 weeks, and every 3 months thereafter for 2 years, to obtain best objective response by Response Evaluation Criteria in Solid Tumors (RECIST).

\section{TIL production}

The generation of 'young' TIL was based on established protocols. ${ }^{23}{ }^{26}$ In brief, TIL isolation and expansion was started by generation of a single cell suspension by enzymatic digestion of the resected metastatic tumor material obtained by surgery, using collagenase type IV (Sigma Aldrich) and Pulmozyme (Roche). Resulting cell suspensions were cultured in the presence of $6000 \mathrm{IU} / \mathrm{mL}$ IL-2 (Proleukin, Novartis) for 2-4 weeks until a minimum of $1 \times 10^{8}$ cells was achieved. At this point, TIL were either frozen (for logistic reasons) or directly expanded using an REP. In this process, $35 \times 10^{6}$ CD3 positive $\mathrm{T}$ cells were cultured in $50 \%$ Roswell Park Memorial Institute (RPMI) / 50\% AIM-V medium in the presence of 3000 $\mathrm{IU} / \mathrm{mL}$ IL-2, $30 \mathrm{ng} / \mathrm{mL}$ anti-CD3 (OKT-3, MACS GMP CD3 pure, Miltenyi) and irradiated autologous PBMCs, at a 200:1 feeder cell: TIL ratio. Cultures were initiated in 35 T175 culture flasks and continued in a WAVE bioreactor from day 6 onwards. ${ }^{26}$ At day 14, TIL were harvested and concentrated by centrifugation, followed by a wash step with PBS containing 5\% human serum albumin. TIL were then suspended in a physiological saline solution, supplemented with $2.5 \%$ human serum albumin (Sanquin Blood Supply) and $300 \mathrm{IU} / \mathrm{mL}$ IL-2, as the final infusion formulation. 
TIL manufacturing was performed in a class B cleanroom (GMP EU Annex 1) with strict air pressure hierarchy for combining aseptic conditions with containment. Handling of product with direct exposure to the environment was performed in a class A (GMP EU Annex 1) biohazard laminar down-flow cabinet with a class $B$ background. Performance of the cleanroom facility was monitored by measurement of the levels of microorganisms and particles both at operating state and at rest state. In addition, aseptic handling was validated by periodic medium fills. Final quality control of TIL products consisted of cell number (between $5 \times 10^{9}$ and $2 \times 10^{11}$ ), viability $(>70 \%)$, sterility, and the absence of tumor cells above level of detection. Cell number and viability was determined by visual counting using a hemacytometer under a microscope, according to Ph.Eur. 2.7.29. Sterility was confirmed using the BacT/ALERT system (Biomerieux), which was validated according to Ph. Eur. 2.6.27. The absence of detectable residual tumor cells was determined in a cytospin by immunohistochemical staining for the tumor markers MART-1, gp-100 and S100. The sensitivity of the test was determined to be $0.2 \%$ of tumor cells within a TIL population.

\section{Phenotypic characterization}

A small fraction of TIL infusion products was cryopreserved for subsequent phenotypic characterization by flow cytometry. In brief, after thawing, cells were rested for 24 hours, and then stained using antibodies directed against: CD3 (clone SK7), CCR7 (clone 150503), CD28 (clone CD28.2), CD62L (clone SK11) (all BD Biosciences), CD4 (clone S3.5), CD8 (clone 3B5), CD45RA (clone MEM-56), CD27 (clone CLB-27/1) (all Invitrogen) and PD-1 (clone J105, eBioscience). Fixable Violet Dead Cell Stain Kit (Invitrogen) was used to exclude dead cells. Cells were analyzed on a LSR2 SORP flow cytometer (BD Biosciences) and data was processed using Flowjo_V10 software.

\section{Intracellular cytokine release assay}

A total of $2 \times 10^{5}$ TILs were cultured with $2 \times 10^{5}$ cells from tumor digest or tumor cell lines, in the presence of $1 \mu \mathrm{L} /$ $\mathrm{mL}$ Golgiplug (BD Biosciences). After 5 hours of incubation, cells were stained using anti-CD8 antibody (clone SKI, BD Biosciences) and live/dead marker (Fixable Violet Dead Cell Stain Kit, (Invitrogen)). Intracellular cytokine production was detected after fixation and permeabilization with the Cytofix/Cytoperm kit (BD Biosciences) and antibodies against interferon- $\gamma$ (IFN- $\gamma$ ) (clone B27), IL-2 (clone Mq1-17H12), and tumor necrosis factor $\alpha$ (TNF- $\alpha)$ (clone Mab11), all from BD Biosciences. Cells were analyzed on an LSR2 SORP flow cytometer and data were processed using Flowjo_V10 software.

\section{Neoantigen-specific T cell reactivity}

Exome sequencing of tumor and autologous healthy PBMCs was performed to identify tumor specific mutations. DNA libraries were generated using the Illumina
TruSeq DNA library preparation kit. Resulting gDNA libraries were enriched for exonic sequences using the Sure Select Human All Exon $50 \mathrm{Mb}$ Target Enrichment system (Agilent, Cat.no. 5190-2851). RNA was isolated using the RNeasy purification kit (QIAGEN). Poly(A)selected RNA libraries were prepared using the TruSeq RNA library protocol (Illumina, San Diego, California, USA), and resulting libraries were sequenced on an Illumina HiSeq2000 using 75 bp paired-end reads. Reads were aligned (Tophat version 1.3.3 (7)); expression values were calculated as fragments per kilobase of transcript per million mapped reads (FPKM) using cufflinks (V.1.0.2 (8)). Mutations identified in genes with FPKM=0 were excluded from further analysis. Subsequently, all potential 9, 10 and 11mers were filtered using three parameters: (1) likelihood of proteasomal processing (NetChop score $>0.49$ ); (2) predicted binding affinity to patient specific HLA alleles (with the highest ranking 35 peptides included per 100 mutations for each allele, yielding a cut-off of approximately $1700 \mathrm{nM}$ for HLA$A * 0201$ ) using NetMHCpan and (3) A similarity-to-self test predicting the degree of similarity between the mutated epitope and the wild type counterpart. ${ }^{27}$ Predicted peptides were synthesized (LUMC, Leiden, The Netherlands), and major histocompatibility complex (MHC) multimers containing these epitopes were produced by microscale parallel UV-induced peptide exchange reactions. ${ }^{28}{ }^{29}$ Subsequently, TILs and PBMCs from patients were analyzed for reactivity against these predicted neoantigens by a multiplexed peptide MHC multimer staining strategy, as described previously. ${ }^{29}$

For patients 3 and 4, a previously described immortalized B cell platform was also utilized for the detection of neoantigen reactivity. ${ }^{31}$ In brief, peptides that covered the mutant amino acid flanked by nine amino acids on each side were synthesized for each mutation and were loaded onto autologous immortalized B cells. Subsequently, B cells were incubated for 48 hours with sorted $\mathrm{CD} 8^{+}$cells from the TIL product, and recognition of mutant peptides was assessed by Cytokine Bead Array (BD Science). Peptides were first tested in pools of five peptides, that were subsequently deconvoluted using individual peptides. Any hits obtained were subsequently confirmed by MHC multimer-based flow cytometry.

\section{Statistical analysis}

TIL characteristics were compared for responders and non-responders, using the two-tailed Mann-Whitney $\mathrm{U}$ test (SPSS V.22).

\section{RESULTS}

\section{Patient characteristics}

In the period between 2005-2011 and 2004-2014, 13 patients were enrolled in this study. The prolonged follow-up of this patient group renders analysis of potential patient benefit more informative. For all patients enrolled, TIL products were successfully generated. Three 
Table 1 patient characteristics

\begin{tabular}{|c|c|c|c|c|c|c|}
\hline Patient nr & Gender & Age & Prior treatments & Metastatic sites & $\begin{array}{l}\text { Location for tumor } \\
\text { resection }\end{array}$ & $\begin{array}{l}\text { Performance } \\
\text { status }\end{array}$ \\
\hline 1 & M & 67 & DTIC, ipilimumab & $\begin{array}{l}\text { Lung, adrenal, } \\
\text { subcutaneous }\end{array}$ & Subcutaneous & 0 \\
\hline 2 & M & 68 & $\begin{array}{l}\text { DTIC, DC vaccination, } \\
\text { ipilimumab }\end{array}$ & $\begin{array}{l}\text { Lung, LN, spleen, } \\
\text { subcutaneous }\end{array}$ & $\mathrm{LN}$ & 0 \\
\hline 3 & M & 46 & $\begin{array}{l}\text { Vemurafenib, } \\
\text { ipilimumab }\end{array}$ & LN, subcutaneous & Subcutaneous & 0 \\
\hline 4 & $\mathrm{~F}$ & 44 & $\begin{array}{l}\text { DTIC, vemurafenib, } \\
\text { ipilimumab }\end{array}$ & $\begin{array}{l}\text { LN, subcutaneous, soft } \\
\text { tissue }\end{array}$ & Subcutaneous & 0 \\
\hline 5 & $\mathrm{~F}$ & 40 & DTIC, ipilimumab & $\begin{array}{l}\text { Lung, subcutaneous, soft } \\
\text { tissue }\end{array}$ & Subcutaneous & 0 \\
\hline 6 & $\mathrm{~F}$ & 43 & $\begin{array}{l}\text { Temozolamide, IFN- } \alpha \text {, } \\
\text { DC vaccination, }\end{array}$ & LN, long, subcutaneous & Axillary LN & 0 \\
\hline 7 & M & 52 & DTIC, ipilimumab & LN, lung, subcutaneous & LN & 0 \\
\hline \multirow[t]{2}{*}{8} & M & 40 & $\begin{array}{l}\text { Peptide vaccination, } \\
\text { ipilimumab }\end{array}$ & LN, small intestine & Large intestine & 0 \\
\hline & & & & & Mesoduodenum & \\
\hline 9 & $\mathrm{~F}$ & 60 & $\begin{array}{l}\text { Vemurafenib, DTIC, } \\
\text { ipilimumab }\end{array}$ & LN, subcutaneous & $\mathrm{LN}$ & 1 \\
\hline 10 & M & 55 & $\begin{array}{l}\text { DC vaccination, } \\
\text { ipilimumab }\end{array}$ & $\begin{array}{l}\text { LN, subcutaneous, liver, } \\
\text { lung }\end{array}$ & Jejunum & 1 \\
\hline
\end{tabular}

DC, dendritic cell; DTIC, dacarbazine; IFN- $\alpha$, interferon- $\alpha$; LN, lymph node.

patients did not receive the final TIL product because of rapid disease progression. All patients had been heavily pretreated with chemotherapy, immunotherapy and/or targeted therapy (see table 1).

\section{Clinical results}

Out of the 10 treated patients, two achieved a CR, three achieved a partial response (PR), one patient had stable disease for a period of 2 months and four patients showed progressive disease (table 2). None of these patients had received anti-PD-1 therapy, since this was not available during enrollment of this study. Figure $1 \mathrm{~A}$ shows a waterfall plot of best clinical response.

For three patients with an objective response to TIL therapy, the presence of neoantigen-specific $\mathrm{T}$ cell populations in the TIL product and in the peripheral blood compartment was analyzed and related to clinical course.

Patient 3 was a 46-year-old male with BRAF V600E mutation positive melanoma with in-transit metastases, iliac lymph node metastases, liver metastases and an enlarged left-sided adrenal gland. During prior treatment with vemurafenib, this patient experienced complete resolution of the liver metastases, but progression in lymph node metastases. Subsequently, he was treated with ipilimumab but showed progressive disease after four courses. At the time of study enrolment, this patient showed large in-transit metastases in his right upper leg, as well as iliac lymph node metastases. In addition, the patient had an enlarged adrenal gland, but no signs of liver, pulmonary or brain metastases. One of the in-transit metastases was resected for generation of the TIL product, and the patient received $19.6 \times 10^{10} \mathrm{TIL}$, followed by four boluses of HD IL-2. Because of hypotension and low urinary output not responding to fluid challenges, the patient was on the intensive care unit (ICU) for 2 days requiring inotropic agents. He recovered from TIL treatment without any sequelae, and was in CR 12 weeks after TIL infusion. He remains disease free for more than 9 years after TIL therapy (see figure 1B).

Patient 4 was a female of 44 years of age with stage M1b BRAF V600E mutation positive melanoma with metastases in the left axilla and upper lobe of the left lung. Previously, this patient underwent axillary lymph node dissection and excision of the lung metastasis via video-assisted thoracic surgery. Five months later, she developed several subcutaneous metastases and lymphadenopathy in the left supraclavicular region. Treatment with dacarbazine showed progression after two courses, and treatment with ipilimumab showed progression after four courses. Subsequent treatment with vemurafenib led to a PR that lasted 4 months. This patient received $7 \times 10^{10}$ TIL followed by two doses of IL-2. One month after TIL infusion, her disease was in partial remission. In the months thereafter, no further shrinkage of metastases was observed, and all remaining visible lesions (five soft tissue and one lymph 


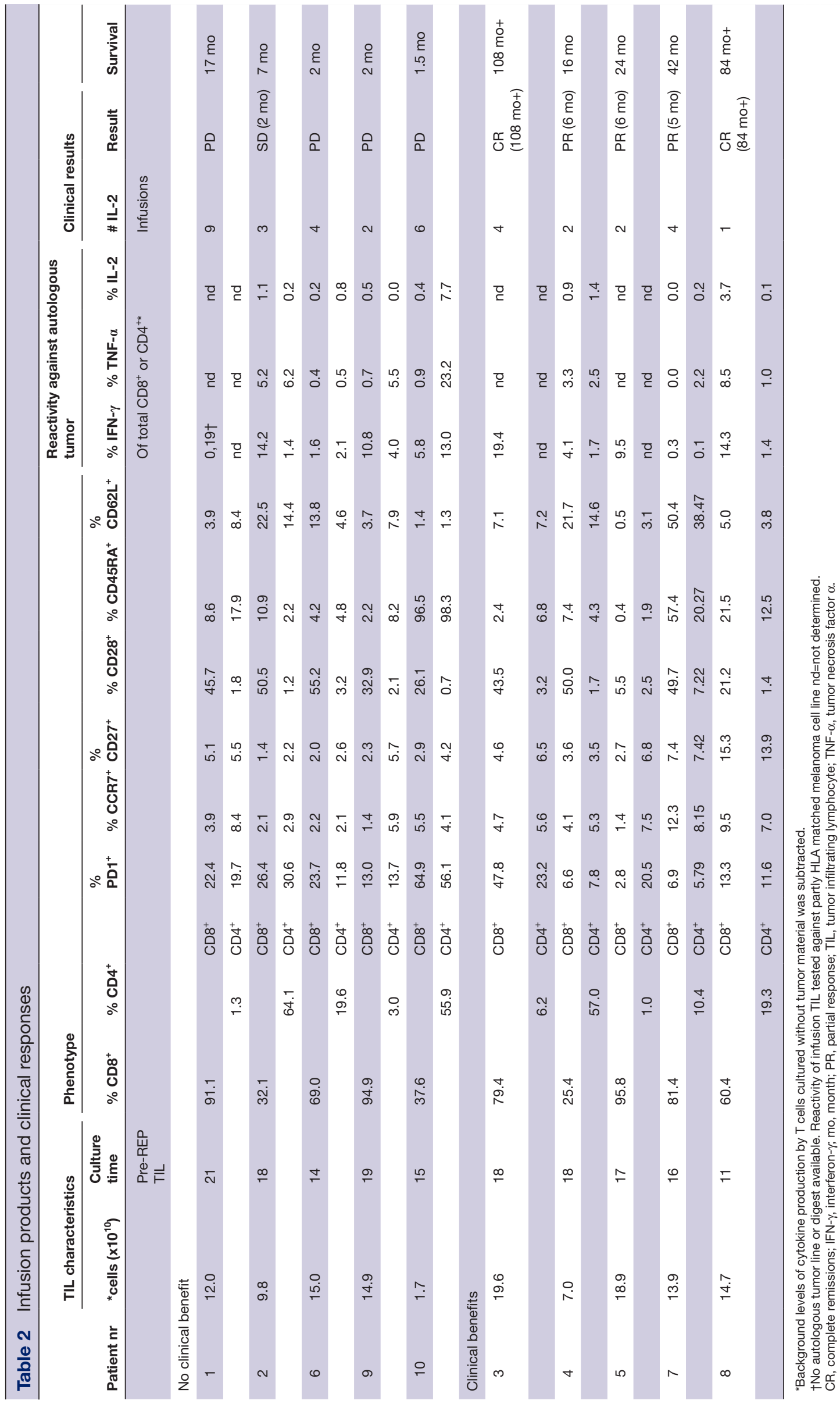


A

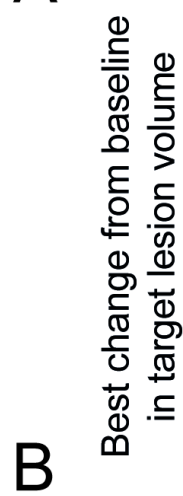

ongoing response

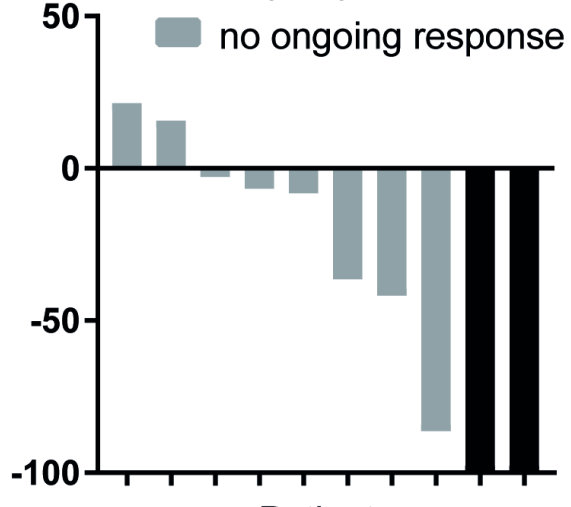

Patients

\section{Prior to TIL}

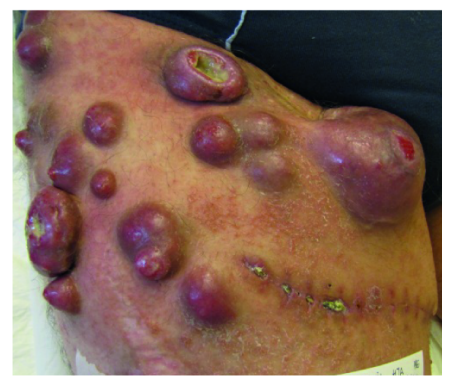

3 wks post TIL

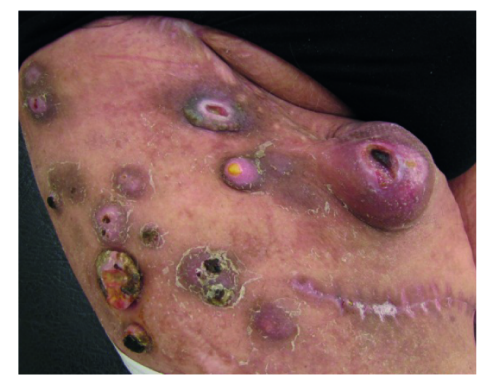

8 wks post TIL

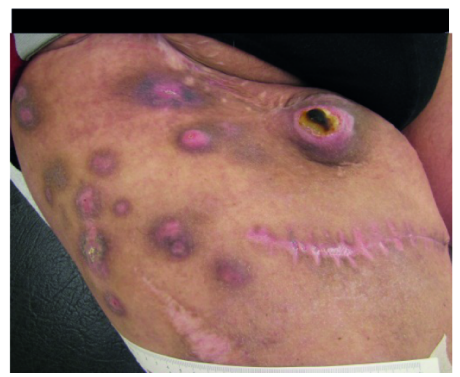

12 wks post TIL

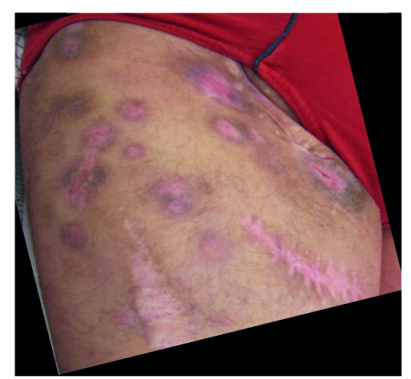

Figure 1 Clinical activity of TIL therapy (A) waterfall plot showing the best overall response for the 10 patients based on CT scans. (B) Photographic pictures of the upper leg of patient 3 who showed an impressive complete response on TIL therapy. The scar identifies the site of tumor resection for TIL isolation. This patient is currently tumor free for 9 years. TIL, tumor infiltrating lymphocyte.

node metastases) were at that point removed by surgery. Unfortunately, her disease recurred 4 months later, for which she again underwent resection. Another 6 months later, the patient had developed multiple brain metastases, and she died 16 months after TIL therapy.

Patient 8 was a 40 -year-old male who was treated elsewhere for stage I and later stage III melanoma. He underwent inguinal lymph node dissection, with a recurrence in the same area 8 months later. These lesions were resected, and 5 months thereafter he developed retroperitoneal lymphadenopathy, for which he again underwent resection. Three months later, he also developed mediastinal lymph node metastases and was treated in an NY-ESO-1 protein vaccine trial. Six months later his disease had progressed. Following two courses of ipilimumab, he developed grade 3 colitis and treatment was halted. Another 6 months later, due to disease progression of peritoneal and mesenteric lymph node metastases, he was referred to our institute for TIL treatment. The patient received $14.7 \times 10^{10} \mathrm{TIL}$, followed by one dose of IL-2. One month after TIL infusion, the mesenteric lymph node metastases showed a PR. Ten months after TIL infusion, the patient was in CR and currently has an ongoing CR for 7 years after therapy.

\section{Toxicity}

All toxicities observed were expected and in line with previous studies using the same treatment regimen. No treatment-related deaths were observed. Median time in hospital for all patients was 16 days (range 12-31). All patients underwent the scheduled lymphodepleting chemotherapy regimen. Anticipated hematological toxicities were seen in all of the treated patients. A grade 4 decrease in neutrophils was seen in all patients. Grade 3-4 decrease in platelets was seen in seven patients $(70 \%)$ and grade 3-4 anemia was observed in three patients (30\%). Patients received platelet and red blood cell transfusions as required based on standard protocols. Patients received on average 2.5 units of platelets (range $0-6$ ) and 2.5 units of red blood cells (range 0-16). TIL infusion was mostly accompanied by low-grade side effects such as dyspnea, fever, nausea and chills. One patient experienced grade 3 dyspnea, possibly due to an allergic reaction, shortly after TIL infusion for which she was treated with budesonide inhalation and additional oxygen (4-5 L/min). Except for chemotherapy related grade 3-4 toxicities, no other grade 3-4 toxicities were observed during, or shortly after TIL infusion. All patients received HD bolus IL-2 (600 000 IU $/ \mathrm{kg}$ ) starting 4 hours after TIL transfusion. A median of 3.5 cycles of HD IL-2 was administered to the patients (range 1-9). None of the patient received all 15 cycles of HD IL-2. All patients experienced febrile neutropenia grade 3-4 during the HD IL-2 period. Other commonly seen toxicities during the HD IL-2 period were chills (eight patients), dyspnea (eight patients), tachycardia 
(six patients) and oliguria (four patients). All patients received Granulocyte colony-stimulating factor (G-CSF) injections once daily $(5 \mu \mathrm{g} / \mathrm{kg}$, with a maximum of 600 $\mu \mathrm{g})$ starting 24 hours after TIL infusion, until neutrophil recovery. Patients received broad-spectrum antibiotics and antipyretic drugs (acetaminophen and occasionally indomethacin), according to local guidelines. Four patients (40\%) had to be admitted to the ICU. Three patients due to oliguria and the need for inotropic agents, and one patient due to the appearance of a pneumothorax, probably caused by the insertion of a subclavian catheter, for which he required chest drain insertion. Most IL-2-related toxicities were transient and resolved within a few days after discontinuation of IL-2. Autoimmune adverse events were seen in one patient (patient 6). This patient possibly developed clinical symptoms reminiscent of Vogt-Koyanagi-Harada-like syndrome with an anterior uveitis and hearing loss several days after TIL infusion. She was treated with prednisone eye-drops with quick resolution of visual complaints. Treatment of patients with a lymphodepleting chemotherapy regimen, followed by the infusion of TIL and HD bolus IL-2 appeared safe, as no therapy-related deaths were seen. Furthermore, all grade 3-4 toxicities were readily managed on the internal medicine ward or ICU, and no long-term side effects were seen.

\section{TIL characteristics}

All TIL products complied with the preset specifications. The average number of cells in the infusion product was $12.8 \times 10^{10}$ (range 1.7-19.6), with an average pre-REP culture time of 16.7 days (range 11-21). The viability of all samples was above 95\%. All cryopreserved infusion products were phenotypically and functionally characterized on coincubation with autologous tumor digest (see table 2 for an extensive overview).

In most infusion products, the majority of cells consisted of $\mathrm{CD}^{+} \mathrm{T}$ cells, with a smaller fraction of $\mathrm{CD} 4^{+} \mathrm{T}$ cells. In all tested samples, in vitro reactivity of both $\mathrm{CD}^{+}$and
$\mathrm{CD}^{+} \mathrm{T}$ cells against autologous tumor digest could be detected at varying levels (with IFN- $\gamma$ levels between $0.3 \%$ and $19 \%$ of total $\mathrm{CD}^{+}$cells). In addition, tumor-induced production of other $\mathrm{T}$ cell effector cytokines, such as TNF- $\alpha$ and IL-2, was noted. The majority of $\mathrm{CD}^{+}$cells were single producers of IFN- $\gamma$, suggesting a more advanced differentiation state. ${ }^{32}$ However, a smaller fraction of $\mathrm{T}$ cells in all patients was shown to produce a combination of multiple effector cytokines in response to tumor cell encounter (see online supplementary figure 1). In previous trials using TIL treatment in melanoma patients, a correlation between TIL culture time, percentage of $\mathrm{CD}^{+}$, percentage of $\mathrm{CD}^{+} / \mathrm{CD} 27^{+}$, number of IL-2 infusions and clinical benefit has been described. ${ }^{192022}$ In the current cohort, no such correlations could be detected potentially due to the small sample size.

\section{Neoantigen-specific T cell reactivity in TIL infusion products}

Having observed $\mathrm{T}$ cell reactivity against tumor cells in all evaluated TIL infusion products, we set out to determine the occurrence of $\mathrm{T}$ cell responses against patient-specific neoantigens, using a previously developed pipeline. ${ }^{730} \mathrm{In}$ order to be able to relate expansion of neoantigen-specific $\mathrm{T}$ cells to tumor regression, the infusion products of the three patients who either developed a PR (patient 4) or CR on TIL therapy (patient 3 and 8 ) were analyzed. In all three patients, neoantigen-specific $\mathrm{T}$ cell reactivity could be detected in the TIL product (see table 3), but with a major variation in response breadth and magnitude. Specifically, in patient $3, \mathrm{CD}^{+} \mathrm{T}$ cell reactivity against five different neoantigens was observed, with $\mathrm{CD}^{+} \mathrm{T}$ cells directed against neoantigen RBM12 $\mathrm{S}>\mathrm{L}$ forming a major component (29\% of total $\mathrm{CD}^{+}$cells) of the therapeutic cell product. While patient 8 also experienced prolonged and ongoing clinical benefit of TIL therapy, T cell reactivity in the cell product used for therapy was only detected for a single predicted neoantigen, and this response was of a low magnitude $\left(0.17 \%\right.$ of total $\mathrm{CD} 8^{+} \mathrm{T}$ cells $)$. Notably, total tumor reactivity of this TIL product, as measured

Table 3 Characteristics of identified neoantigen-specific T cell responses in TIL infusion products

\begin{tabular}{|c|c|c|c|c|c|}
\hline & Gene mutation & WT peptide & Mutant peptide & $\begin{array}{l}\text { Frequency }(\% \\
\text { multimer }^{+} \mathrm{CD} 8^{+} \\
\mathrm{T}^{+} \text {cells of total } \\
\text { CD8 }^{+} \mathrm{T} \text { cells) }\end{array}$ & $\begin{array}{l}\text { Restriction } \\
\text { element }\end{array}$ \\
\hline \multirow[t]{5}{*}{ Patient 3} & $\mathrm{RBM} 12_{\mathrm{S}>\mathrm{L}}$ & SPHEAGFCV & LPHEAGFCV & 29.00 & HLA-B*51:01 \\
\hline & VARS $_{T>M}$ & EVADEATGAL & EVADEAMGAL & 0.096 & HLA-A*25:01 \\
\hline & MYLK $_{G>V}$ & EVFPEDTGTY & EVFPEDTVTY & 1.90 & HLA-A*25:01 \\
\hline & $\mathrm{LRP}_{\mathrm{T}>\mathrm{S}}$ & LTAARPSQTVL & LTAARPSQSVL & 0.039 & HLA-A*25:01 \\
\hline & $W D R 1_{N>K}$ & DSFAGKGHTN & DSFAGKGHTK & 0.50 & HLA-A*68:01 \\
\hline \multirow[t]{3}{*}{ Patient 4} & TTC37 A>V & YLDGKAVDY & YLDGKVVDY & 1.14 & HLA-A*01:01 \\
\hline & ENTPD4 $P>L$ & ATDTNNPNVNY & ATDTNNLNVNY & 3.35 & HLA-A*01:01 \\
\hline & MAB21L1 $v>M$ & LRIRDRYVV & LRIRDRYVM & 0.007 & HLA-B*08:01 \\
\hline Patient 8 & RAD51AP1-002 $s>F$ & KVKSPVEKK & KVKFPVEKK & 0.195 & HLA-A*03:01 \\
\hline
\end{tabular}

TIL, tumor infiltrating lymphocyte. 
A Patient 3

RBM12 S>L
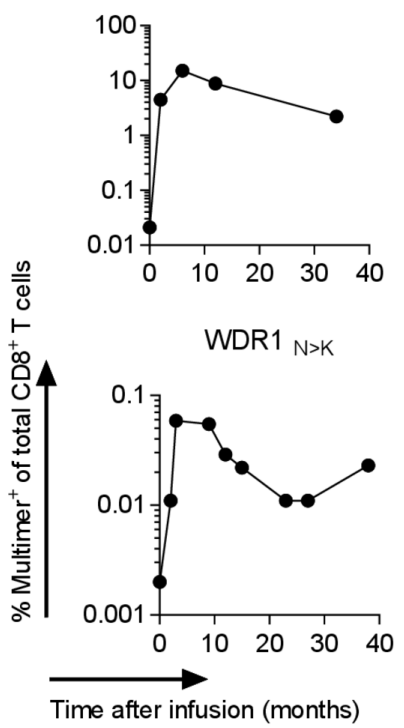

B Patient 4

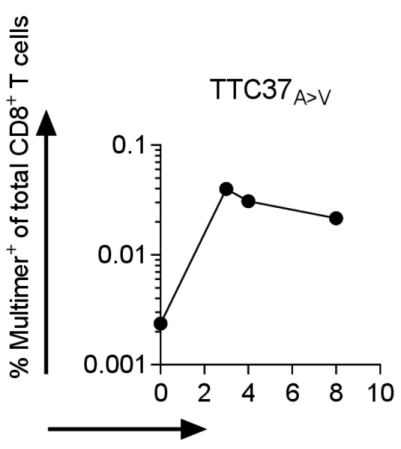

Time after infusion (months)
VARS $_{T>M}$

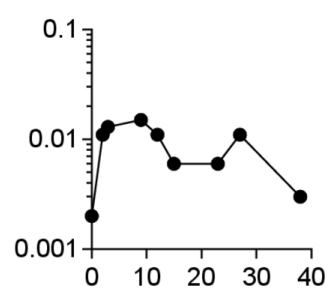

MYLK $_{G>V}$

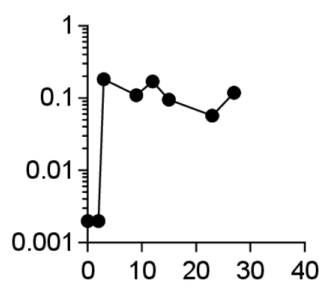

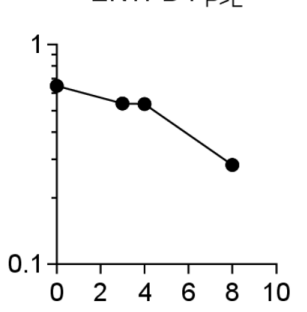

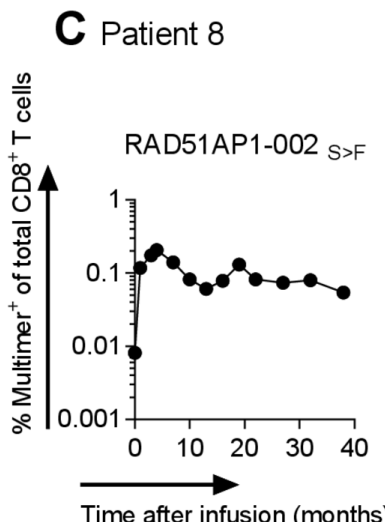

Figure 2 Kinetics of neoantigen-specific CD8 ${ }^{+} \mathrm{T}$ cell responses in TIL responders Kinetics of neoantigen-specific CD8 ${ }^{+} \mathrm{T}$ cell responses in peripheral blood of patient 3 (A), 4 (B) and 8 (C). T cell responses against the indicated epitopes were first identified in infusion products by MHC multimer screening and subsequently followed in peripheral blood using the same technology. HC, major histocompatibility complex; TIL, tumor infiltrating lymphocyte.

using functional assays, was substantially higher (15\% of $\mathrm{CD}^{+} \mathrm{T}$ cells, see table 2$)$, either indicating the existence of neoantigen-specific $\mathrm{T}$ cell reactivities that were missed by the pipeline used, or indicating that reactivities against non-mutant antigens may form a major component in this particular patient. Note that MHC multimer screens were performed for six out of six HLA-A alleles, five out of six HLA-B alleles and none of the HLA-C alleles expressed by these patients. In patient 4 , three unique neoantigenspecific $\mathrm{T}$ cell populations could be detected in the infusion product with frequencies of $0.007,1.14 \%$ and $3.35 \%$ of total $\mathrm{CD} 8^{+}$cells. The infusion product of this patient also contained neoantigen-specific $\mathrm{CD} 4^{+} \mathrm{T}$ cells, with a frequency of $3.8 \%$ of $\mathrm{CD} 4^{+} \mathrm{T}$ cells against a single neoantigen, as reported previously. ${ }^{30}$

\section{Neoantigen-reactivity in peripheral blood}

Having identified neoantigen-specific $T$ cell populations in the TIL products of all responding patients, we subsequently determined whether engraftment and expansion of the different neoantigen-reactive $\mathrm{T}$ cell pools could be observed (see figure 2 and table 4 ).

Analysis of pretreatment blood samples of the three different patients revealed the presence of only low levels of $\mathrm{T}$ cells against the identified neoantigens in peripheral blood before TIL infusion (Patient 3, RBM12 $\mathrm{S}>\mathrm{L}$ : $0.03 \%$ MHC multimer ${ }^{+}$of $\mathrm{CD}^{+}$, patient 4 , TTC37 ${ }_{\mathrm{A}>\mathrm{V}}$ : $0.002 \%$ MHC multimer ${ }^{+}$of $\mathrm{CD}^{+}$, ENTPD $4_{\mathrm{P}>\mathrm{L}}: 0.6 \%$ MHC multimer ${ }^{+}$of $\mathrm{CD}^{+}$, patient 8, RAD51AP1-002 ${ }_{\mathrm{S}>\mathrm{F}}$ : $0.008 \%$ MHC multimer $^{+}$of $\mathrm{CD}^{+}$). The other neoantigenspecific $\mathrm{T}$ cell populations present in TIL products were not detected in the pre-treatment blood compartment (detection level: $0.002 \% \mathrm{MHC}$ multimer ${ }^{+}$of $\mathrm{CD}^{+} \mathrm{T}$ cells and at least 10 events in flow cytometry plot).

To understand to what extent TIL therapy boosted the capacity of the peripheral blood compartment to recognize this set of neoantigens, peripheral blood samples were monitored at different time points post-therapy. Strikingly, for six out of the nine neoantigen-specific T cell 
Table 4 Fold expansion of identified neoantigen-specific T cell responses in peripheral blood

\begin{tabular}{|c|c|c|c|}
\hline & Gene mutation & $\begin{array}{l}\text { Peak of neoantigen-specific T cell } \\
\text { frequency in blood (months after TIL } \\
\text { infusion) }\end{array}$ & $\begin{array}{l}\text { Minimal fold expansion } \\
\text { compared with } \\
\text { preinfusion at peak }\end{array}$ \\
\hline \multirow[t]{4}{*}{ Patient 3} & $R B M 12_{S>L}$ & 6 & 750 \\
\hline & VARS $_{T>M}$ & 9 & $8^{*}$ \\
\hline & MYLK $_{G>V}$ & 3 & $92^{*}$ \\
\hline & $\mathrm{LRP}_{\mathrm{T}>\mathrm{S}}$ & Not detected in blood & Not applicable \\
\hline \multirow[t]{3}{*}{ Patient 4} & TTC37 $\mathrm{A>V}$ & No expansion observed & 1 \\
\hline & ENTPD4 ${ }_{P>L}$ & 3 & 17 \\
\hline & MAB21L1 $_{V>M}$ & Not detected in blood & Not applicable \\
\hline Patient 8 & RAD51AP1-002 ${ }_{S>F}$ & 4 & 25 \\
\hline
\end{tabular}

${ }^{*}$ Calculated using the detection limit of the assay for the preinfusion time point, which is $0.002 \% \mathrm{MHC}$ multimer ${ }^{+}$of total $\mathrm{CD}^{+} \mathrm{T}^{\mathrm{C}}$ cells. $\mathrm{MHC}$, major histocompatibility complex; TIL, tumor infiltrating lymphocyte.

reactivities analyzed, a substantial increase in frequency in post infusion blood samples could be observed. One of the nine responses, specific for the ENTPD4 ${ }_{\mathrm{P}>\mathrm{L}}$ mutation identified in patient 4 , remained stable in blood on therapy. The other two neoantigen-specific T cell reactivities, which were both found at very low levels in the infusion products (patient 4, MAB21L1 $1_{v>m}$ and patient 3, LRP3 ${ }_{\mathrm{T}>\mathrm{S}}$ ), could not be detected above background in peripheral blood samples before or after therapy, precluding any assessment of change in frequency. Table 4 provides an overview of the minimum fold expansion in individual neoantigen-specific $\mathrm{T}$ cell responses prior to therapy and post-therapy.

The neoantigen-specific $\mathrm{T}$ cell population directed against RBM12 ${ }_{\mathrm{S}>\mathrm{L}}$, which had the highest frequency in the TIL infusion samples, also showed the highest expansion in the blood. At 6 months post-therapy, a 750-fold expansion compared with pre-TIL infusion was observed. $T$ cells reactive against the $\mathrm{RBM} 12 \mathrm{~S}>\mathrm{L}$ epitope remained detectable up to 34 months postinfusion (the last time point analyzed), at a frequency of $2.2 \%$. The observed expansion and contraction of this neoantigen-specific $\mathrm{T}$ cell response that coincided with clinical response provides indirect temporal evidence for a role of this $\mathrm{T}$ cell pool in cancer regression. The low level presence of these neoantigen-specific $\mathrm{T}$ cells almost 3 years after development of a complete clinical response may both reflect the formation of $\mathrm{T}$ cell memory, or the development of an equilibrium between a clinically undetectable reservoir of tumor cells and an active $\mathrm{T}$ cell response.

In patient 4 (PR), analysis of the kinetics of neoantigenspecific $\mathrm{T}$ cell responses post-TIL therapy demonstrates that $\mathrm{T}$ cell reactivities against different neoantigens in an individual patient can show a disparate behavior. In this patient, one neoantigen-specific $\mathrm{CD}^{+} \mathrm{T}$ cell response showed a substantial increase (17 fold) on therapy, whereas a second neoantigen-specific $\mathrm{T}$ cell response remained stable on TIL infusion (see figure 2B). Both responses were already detectable before infusion at low frequencies and could be detected at the latest time point (8 months post-therapy) for which material was available. Interestingly, the previously reported CD4 response in patient 4 remained detectable in blood during this 8 month follow-up as well.

The single neoantigen-specific $\mathrm{CD}^{+} \mathrm{T}$ cell response that was observed in the TIL product from patient 8 (CR) was detectable at a very low frequency in the peripheral blood compartment prior to TIL infusion $(0.008 \% \mathrm{MHC}$ multimer $^{+}$of total CD $8^{+} \mathrm{T}$ cells). Following TIL infusion, this $\mathrm{T}$ cell response increased over time and peaked at 4 months after infusion at a 25-fold higher frequency than pretreatment levels. In the subsequent years, this $\mathrm{T}$ cell response remained detectable in blood at $0.05 \%$, revealing an expansion and contraction pattern similar to that observed in patient 3 .

Collectively, these results demonstrate that for the majority of neoantigen-specific $\mathrm{T}$ cell responses (six out of nine) analyzed, TIL therapy leads to a sizeable increase in $\mathrm{T}$ cell reactivity in the peripheral blood compartment, with an observed increase in neoantigen-specific $\mathrm{T}$ cell numbers between 8-fold and 750-fold.

\section{Phenotype of neoantigen-specific T cells}

Phenotypic analysis of the neoantigen-specific $\mathrm{T}$ cells populations in the infusion product demonstrated low CCR7 expression and a mixture of CD45RA negative and positive cells, indicating a mix of effector and effector memory like $\mathrm{T}$ cells. Remarkably, this distribution of phenotypes did not change over time, toward a more central memory phenotype, when this neoantigenspecific population was followed in blood over several months postinfusion (online supplementary figure 2A,B and $3 \mathrm{~A}, \mathrm{~B}$, data presented for patient 4 and 8 ). In addition, we observed that PD-1 expression, as a marker for $\mathrm{T}$ cell activation, was higher on the neoantigen-specific $\mathrm{T}$ cell population as compared with total bulk $\mathrm{CD} 8^{+}$in 
peripheral blood, and its expression did not change over time when looking at the neoantigen-specific population in blood after infusion (online supplementary figures 2C and $3 \mathrm{C}$, data presented for patient 4 and 8 ).

\section{CONCLUSION AND DISCUSSION}

In accordance with the results obtained by other clinical sites for TIL therapy, ${ }^{21-24}$ we demonstrate an overall response rate of $50 \%$, including a $20 \%$ durable CR rate in this small clinical trial. It should be stressed that these patients were enrolled before the introduction of antiPD-1 therapy. The responses on anti-PD-1 failure are reported to be somewhat lower (around 30\%-40\%). ${ }^{25}$ Successful completion of this small-scale feasibility study, plus the encouraging clinical signal seen has led to the initiation of an ongoing randomized phase III multicentre trial together with the CCIT research group in Copenhagen, Denmark, in which TIL therapy is compared with standard of care ipilimumab for metastatic melanoma patients who progress after anti-PD-1 therapy (NCT02278887).

The executing of this small feasibility study was successful at the NKI, due to the presence of highly specialized staff (including solid knowledge on TIL production and GMP), a very close interplay between GMP facility and the clinic (which is logistically required when using fresh infusion products) and the strong interaction between basic immunologists and clinical scientists (required for the translational research presented here).

Neoantigen-specific $T$ cell reactivity could be observed in all the infusion products analyzed. In six out of nine cases, neoantigen-specific T cell responses in the peripheral blood compartment were directly increased on TIL infusion. The frequency of those neoantigen-specific $\mathrm{T}$ cells responses peaked between 3 and 9 months. Of note, in the two CR patients in whom neoantigen-specific $\mathrm{T}$ cell responses were analyzed for years following therapy, persistence of neoantigen-specific $\mathrm{T}$ cell responses until the latest time point analyzed, almost 3 years after infusion, was observed. These data suggest that while many of the cells present in TIL products may be terminally differentiated, a subset appears present that has the capacity for long-term survival and renewal. Importantly, the effector to effector-memory phenotype of these neoantigenreactive T cells did not change over time to a more centralmemory phenotype, neither did expression of PD-1 on these circulating $\mathrm{T}$ cells go down. The reason for this is currently not clear. One could speculate that either these cells are imprinted through an epigenetic program not to be able to switch to a central-memory phenotype, typically observed for virus-specific cells following an acute viral infection, ${ }^{33}$ or these cells are still 'seeing' their cognate antigen (at a very low level). The latter could only be explained if an equilibrium exists between residual tumor and these immune cells. We consider this option, with a follow-up time of several years very unlikely.
The contribution of the neoantigen-specific $\mathrm{T}$ cell populations detected within the TIL infusion products to the observed clinical responses remains to be determined. An argument in favor of a significant contribution of these neoantigen-specific $\mathrm{T}$ cells within TIL is the observed strong in vivo expansion of six out of nine analyzed neoantigen-specific $\mathrm{T}$ cell responses. Further support for this model comes from the observed relationship between tumor mutational burden and response to TIL therapy,${ }^{34}$ and the early clinical data with neoantigenenriched TIL infusion products. ${ }^{35}$

On failure of anti-PD-1 therapy, which occurs in the majority of patients, treatment options are relatively limited, especially in patients with BRAF wild-type disease. Prospective data on the use of ipilimumab following antiPD-1 failure show an objective response rate (ORR) of 10\%-15\%. A recent single-arm phase II trial of TIL therapy in patients who progressed on anti-PD-1 showed a promising $38 \%$ ORR, ${ }^{25}$ although with little information on the durability of the response, and these data appear in line with results from other TIL trials in refractory patients, including patients progressing on anti-PD$1 .{ }^{36}{ }^{37}$ In this clinical landscape, a formal comparison of TIL therapy against ipilimumab in anti-PD-1 refractory patients appears warranted.

\section{Author affiliations}

${ }^{1}$ BioTherapeutics Unit, Netherlands Cancer Institute, Amsterdam, The Netherlands ${ }^{2}$ Division of Molecular Oncology and Immunology, Netherlands Cancer Institute, Amsterdam, The Netherlands

${ }^{3}$ AIMM Therapeutics, Amsterdam, The Netherlands

${ }^{4}$ Experimental Immunology, Amsterdam University Medical Centres, Amsterdam, Noord-Holland, The Netherlands

${ }^{5}$ Department of Medical Oncology, Netherlands Cancer Institute, Amsterdam, The Netherlands

${ }^{6}$ Department of Surgery, Leiden Universitair Medisch Centrum, Leiden, Zuid-Holland, The Netherlands

${ }^{7}$ Surgical Oncology, Antoni van Leeuwenhoek Nederlands Kanker Instituut, Amsterdam, The Netherlands

${ }^{8}$ Dutch Institute for Clinical Auditing, Leiden, The Netherlands

${ }^{9}$ Department of Biometrics, Netherlands Cancer Institute, Amsterdam, The Netherlands

${ }^{10}$ Department of Medical Oncology, Netherlands Cancer Institute, Amsterdam, Noord-Holland, The Netherlands

${ }^{11}$ Department of Pharmacy \& Pharmacology, Netherlands Cancer Institute, Amsterdam, The Netherlands

${ }^{12}$ Division of Pharmacoepidemiology and Clinical Pharmacology, Utrecht University Department of Pharmaceutical Sciences, Utrecht, Utrecht, The Netherlands

${ }^{13}$ Oncode Institute, Utrecht, The Netherlands

Acknowledgements We would like to kindly acknowledge the Department of Surgical Oncology and nursing staff of floor $4 \mathrm{~B}$ of the Netherlands Cancer Institute for excellent patient care and crucial support. In addition, the Quality Assurance and Quality Control departments of the Hospital Pharmacy, the Department of Clinical Chemistry and the Department of Pathology of the Netherlands Cancer Institute are kindly acknowledged for their invaluable support of this multidisciplinary trial.

Contributors All authors participated in study supervision; acquisition, TIL production, patient care, analysis or interpretation of data; and critical revisions of the manuscript for important intellectual content.

Funding This study was supported by the NKI-AVL.

Competing interests $\mathrm{JH}$ has a research collaboration with BMS, MSD, Novartis and BionTech USA. JH serves as an advisor or consultant for: AIMM therapeutics, AZ, Amgen, Achilles TX, Bayer, BMS, GSK, Ipsen, Immunocore, MSD, Merck 
Serono, BionTech USA, Neogene Therapeutics, Novartis, Pfizer, Roche/Genentech, Sanofi, Seattle Genetics, Third Rock Ventures and Vaximm. TNS has a research collaboration with Merck KGaA. TNS serves as an adivisor or consultant for: Adaptive Biotechnologies, AIMM Therapeutics, Allogene Therapeutics, Merus, BionTech USA and Scenic Biotech. TNS is a stockholder of AIMM Therapeutics, Allogene Therapeutics, Merus, Neogene Therapeutics, BionTech USA and Scenic Biotech. AIMM Therapeutics holds IP to immortalize human B cells and the AT1412 antibody. HS and RS are employees of AIMM Therapeutics. RS and HS are stockholders of AIMM Therapeutics. The retroviral vectors containing BCL-6 and $\mathrm{BCl}-\mathrm{XL}$ have been generated by a for-profit company, AIMM Therapeutics, which makes the plasmids available. Obtaining the plasmids requires an MTA that includes financial obligations.

\section{Patient consent for publication Not required.}

Ethics approval This study was approved by the national medical ethical committee (CCMO) and conducted in accordance with the Declaration of Helsinki and Good Clinical Practice.

Provenance and peer review Not commissioned; externally peer reviewed.

Data availability statement Data are available on reasonable request. Data can be requested by contacting authors.

Open access This is an open access article distributed in accordance with the Creative Commons Attribution Non Commercial (CC BY-NC 4.0) license, which permits others to distribute, remix, adapt, build upon this work non-commercially, and license their derivative works on different terms, provided the original work is properly cited, appropriate credit is given, any changes made indicated, and the use is non-commercial. See http://creativecommons.org/licenses/by-nc/4.0/.

\section{ORCID iD}

Joost H van den Berg http://orcid.org/0000-0002-4928-8397

\section{REFERENCES}

1 Azimi F, Scolyer RA, Rumcheva P, et al. Tumor-Infiltrating lymphocyte grade is an independent predictor of sentinel lymph node status and survival in patients with cutaneous melanoma. J Clin Oncol 2012;30:2678-83.

2 Kluger HM, Zito CR, Barr ML, et al. Characterization of PDL1 expression and associated T-cell infiltrates in metastatic melanoma samples from variable anatomic sites. Clin Cancer Res 2015;21:3052-60.

3 Kvistborg P, Shu CJ, Heemskerk B, et al. TIL therapy broadens the tumor-reactive CD8(+) T cell compartment in melanoma patients. Oncoimmunology 2012;1:409-18.

4 Coulie PG, Brichard V, Van Pel A, et al. A new gene coding for a differentiation antigen recognized by autologous cytolytic $\mathrm{T}$ lymphocytes on HLA-A2 melanomas. J Exp Med 1994;180:35-42.

5 Kawakami Y, Eliyahu S, Delgado $\mathrm{CH}$, et al. Identification of a human melanoma antigen recognized by tumor-infiltrating lymphocytes associated with in vivo tumor rejection. Proc Natl Acad Sci U S A 1994;91:6458-62.

6 Chomez P, De Backer O, Bertrand M, et al. An overview of the MAGE gene family with the identification of all human members of the family. Cancer Res 2001;61:5544-51.

7 van Rooij N, van Buuren MM, Philips D, et al. Tumor exome analysis reveals neoantigen-specific T-cell reactivity in an ipilimumabresponsive melanoma. J Clin Oncol 2013;31:e439-42.

8 Lu Y-C, Yao X, Li YF, et al. Mutated PPP1R3B is recognized by T cells used to treat a melanoma patient who experienced a durable complete tumor regression. J Immunol 2013;190:6034-42.

9 Robbins PF, Lu Y-C, El-Gamil M, et al. Mining exomic sequencing data to identify mutated antigens recognized by adoptively transferred tumor-reactive T cells. Nat Med 2013;19:747-52.

10 Alexandrov LB, Nik-Zainal S, Wedge DC, et al. Signatures of mutational processes in human cancer. Nature 2013;500:415-21.

11 Le DT, Uram JN, Wang $\mathrm{H}$, et al. Pd-1 blockade in tumors with mismatch-repair deficiency. N Engl J Med 2015;372:2509-20.

12 Rizvi NA, Mazières J, Planchard D, et al. Activity and safety of nivolumab, an anti-PD-1 immune checkpoint inhibitor, for patients with advanced, refractory squamous non-small-cell lung cancer (CheckMate 063): a phase 2, single-arm trial. Lancet Oncol 2015;16:257-65

13 Hodi FS, O'Day SJ, McDermott DF, et al. Improved survival with ipilimumab in patients with metastatic melanoma. $N$ Engl J Med 2010;363:711-23.
14 Homet Moreno B, Parisi G, Robert L, et al. Anti-Pd-1 therapy in melanoma. Semin Oncol 2015;42:466-73.

15 Larkin J, Chiarion-Sileni V, Gonzalez R, et al. Combined nivolumab and ipilimumab or monotherapy in untreated melanoma. N Engl J Med 2015;373:23-34.

16 Larkin J, Chiarion-Sileni V, Gonzalez R, et al. Five-Year survival with combined nivolumab and ipilimumab in advanced melanoma. $N$ Engl J Med 2019;381:1535-46.

17 Rosenberg SA, Dudley ME. Adoptive cell therapy for the treatment of patients with metastatic melanoma. Curr Opin Immunol 2009;21): :233-40.

18 Besser MJ, Shapira-Frommer R, Treves AJ, et al. Clinical responses in a phase II study using adoptive transfer of short-term cultured tumor infiltration lymphocytes in metastatic melanoma patients. Clin Cancer Res 2010;16:2646-55.

19 Itzhaki O, Hovav E, Ziporen Y, et al. Establishment and largescale expansion of minimally cultured "young" tumor infiltrating lymphocytes for adoptive transfer therapy. J Immunother 2011;34:212-20.

20 Rosenberg SA, Yang JC, Sherry RM, et al. Durable complete responses in heavily pretreated patients with metastatic melanoma using T-cell transfer immunotherapy. Clin Cancer Res 2011;17:4550-7.

21 Dudley ME, Wunderlich JR, Yang JC, et al. Adoptive cell transfer therapy following non-myeloablative but lymphodepleting chemotherapy for the treatment of patients with refractory metastatic melanoma. J Clin Oncol 2005;23:2346-57.

22 Radvanyi LG, Bernatchez C, Zhang M, et al. Specific lymphocyte subsets predict response to adoptive cell therapy using expanded autologous tumor-infiltrating lymphocytes in metastatic melanoma patients. Clin Cancer Res 2012;18:6758-70.

23 Besser MJ, Shapira-Frommer R, Itzhaki O, et al. Adoptive transfer of tumor-infiltrating lymphocytes in patients with metastatic melanoma: intent-to-treat analysis and efficacy after failure to prior immunotherapies. Clin Cancer Res 2013;19:4792-800.

24 Andersen R, Donia M, Ellebaek E, et al. Long-Lasting complete responses in patients with metastatic melanoma after adoptive cell therapy with tumor-infiltrating lymphocytes and an attenuated IL2 regimen. Clin Cancer Res 2016;22:3734-45.

25 Sarnaik A, Khushalani NI, Chesney JA, et al. Safety and efficacy of cryopreserved autologous tumor infiltrating lymphocyte therapy (LN-144, lifileucel) in advanced metastatic melanoma patients who progressed on multiple prior therapies including anti-PD-1. JCO 2019;37:2518.

26 Donia M, Larsen SM, Met O, et al. Simplified protocol for clinicalgrade tumor-infiltrating lymphocyte manufacturing with use of the wave bioreactor. Cytotherapy 2014;16:1117-20.

27 Calis JJA, de Boer RJ, Keșmir C. Degenerate T-cell recognition of peptides on $\mathrm{MHC}$ molecules creates large holes in the T-cell repertoire. PLoS Comput Biol 2012;8:e1002412.

28 Toebes M, Rodenko B, Ovaa H, et al. Generation of peptide MHC class I monomers and multimers through ligand exchange. Curr Protoc Immunol 2009; Chapter 18:Unit 18.16.

29 Hadrup SR, Bakker AH, Shu CJ, et al. Parallel detection of antigenspecific T-cell responses by multidimensional encoding of $\mathrm{MHC}$ multimers. Nat Methods 2009;6:520-6.

30 Linnemann C, van Buuren MM, Bies L, et al. High-Throughput epitope discovery reveals frequent recognition of neo-antigens by CD4+ T cells in human melanoma. Nat Med 2015;21:81-5.

31 Kwakkenbos MJ, Diehl SA, Yasuda E, et al. Generation of stable monoclonal antibody-producing $B$ cell receptor-positive human memory B cells by genetic programming. Nat Med 2010;16:123-8.

32 Klebanoff CA, Gattinoni L, Restifo NP. CD8+ T-cell memory in tumor immunology and immunotherapy. Immunol Rev 2006;211:214-24.

33 Delgoffe GM, Powell JD. Feeding an Army: the metabolism of T cells in activation, anergy, and exhaustion. Mol Immunol 2015;68:492-6.

34 Tran E, Turcotte S, Gros A, et al. Cancer immunotherapy based on mutation-specific CD4+ T cells in a patient with epithelial cancer. Science 2014;344:641-5.

35 Lauss M, Donia M, Harbst K, et al. Mutational and putative neoantigen load predict clinical benefit of adoptive T cell therapy in melanoma. Nat Commun 2017;8:1738.

36 Andersen R, Borch TH, Draghi A, et al. T cells isolated from patients with checkpoint inhibitor-resistant melanoma are functional and can mediate tumor regression. Ann Oncol 2018;29:1575-81.

37 Forget M-A, Haymaker C, Hess KR, et al. Prospective analysis of adoptive TIL therapy in patients with metastatic melanoma: response, impact of anti-CTLA4, and biomarkers to predict clinical outcome. Clin Cancer Res 2018;24:4416-28. 